\title{
Regulation of deoxynucleotide metabolism in cancer: novel mechanisms and therapeutic implications
}

\author{
Rebecca Kohnken ${ }^{1 \dagger}$, Karthik M. Kodigepalli ${ }^{1 \dagger}$ and Li Wu ${ }^{1,2,3^{*}}$
}

\begin{abstract}
Regulation of intracellular deoxynucleoside triphosphate (dNTP) pool is critical to genomic stability and cancer development. Imbalanced dNTP pools can lead to enhanced mutagenesis and cell proliferation resulting in cancer development. Therapeutic agents that target dNTP synthesis and metabolism are commonly used in treatment of several types of cancer. Despite several studies, the molecular mechanisms that regulate the intracellular dNTP levels and maintain their homeostasis are not completely understood. The discovery of SAMHD1 as the first mammalian dNTP triphosphohydrolase provided new insight into the mechanisms of dNTP regulation. SAMHD1 maintains the homeostatic dNTP levels that regulate DNA replication and damage repair. Recent progress indicates that gene mutations and epigenetic mechanisms lead to downregulation of SAMHD1 activity or expression in multiple cancers. Impaired SAMHD1 function can cause increased dNTP pool resulting in genomic instability and cell-cycle progression, thereby facilitating cancer cell proliferation. This review summarizes the latest advances in understanding the importance of dNTP metabolism in cancer development and the novel function of SAMHD1 in regulating this process.
\end{abstract}

Keywords: Intracellular dNTP, Regulation, Cancer, Cell proliferation, SAMHD1

\section{Introduction}

Balanced levels of intracellular dNTPs, the building blocks of DNA, are critical in maintaining the genomic integrity of cells. While a reduction or imbalance in dNTPs is known to result in genotoxicity and increased mutagenesis, an increase in dNTPs often results in uncontrolled DNA replication with reduced fidelity that can contribute to cancer development $[1,2]$. Indeed during DNA replication, an unequal and abnormal increase in concentrations of specific dNTPs may result in reduced fidelity $[1,2]$. Many types of cancer cells demonstrate impairment of intracellular dNTP homeostasis, which supports rapid cellular proliferation, enhanced mutagenesis, and contributes to dysregulation of the cell cycle [3]. Nucleotide metabolism, therefore, is a common therapeutic target used

\footnotetext{
* Correspondence: wu.840@osu.edu

${ }^{\dagger}$ Equal contributors

'Center for Retrovirus Research, Department of Veterinary Biosciences, The

Ohio State University, 1900 Coffey Road, Columbus, OH 43210, USA

${ }^{2}$ Department of Microbial Infection and Immunity, The Ohio State University,

Columbus, OH 43210, USA

Full list of author information is available at the end of the article
}

in the treatment of many types of cancer. Nucleoside analogs as well as enzyme inhibitors aim to disrupt the synthetic pathways which result in imbalance of dNTPs in cancer cells [4]. However, the integrated mechanisms, such as anabolic and catabolic pathways, cell cycle control, and DNA repair, which result in impaired dNTP homeostasis in cancer, are incompletely understood.

The intracellular dNTP balance is regulated in part by sterile alpha motif (SAM) and histidine/aspartate (HD)domain containing protein 1 (SAMHD1), the first dNTP triphosphohydrolase (dNTPase) discovered in mammalian cells [5-8]. SAMHD1 acts as the host restriction factor that inhibits human immunodeficiency virus type 1 (HIV-1) infection by reducing intracellular dNTP levels [9]. Human SAMHD1 mutations can cause a severe autoimmune disorder [10], suggesting the importance of its dNTPase function in innate immunity. Mutations of SAMHD1 have been identified in several human cancers [11-19]. SAMHD1 expression is downregulated in many cancers, including leukemia, lymphoma, and solid cancers, such as breast and lung cancer [11,20,21]. Restoration of 
SAMHD1 expression has been reported to reduce cellular proliferation in vitro $[11,21]$. Based on these recent findings, SAMHD1 is proposed to have antiproliferative and tumor suppressive functions in several cancers. Since dNTP metabolism and balance is critical in carcinogenesis, the dNTPase activity of SAMHD1 may mediate its tumor suppressive function. Despite several advancements in in the use of therapeutic nucleoside analogs to target dNTP metabolism, there is yet a need to develop novel and more effective therapeutic strategies in cancer treatment. Further studies to understand the physiological significance of SAMHD1 in cancer can aid in this process.

Although numerous studies have investigated the dNTPase function of SAMHD1 in viral restriction and immune responses, its significance in cancer development and progression has lately been an emerging interest. Functional significance of SAMHD1 and its dNTPase activity in cancer pathophysiology has not yet been reviewed. Here we highlight the importance of dNTP homeostasis in cancer and dNTP regulation by SAMHD1. We also discuss the potential role of SAMHD1 as a tumor suppressor and future studies required to better understand its function for cancer therapeutic development.

\section{Regulation of intracellular dNTPs and its role in cancer Intracellular dNTP synthesis and regulation}

Coordinated synthesis and degradation of dNTPs resulting in a balanced intracellular dNTP pool is critical for numerous cellular processes, such as fidelity of DNA synthesis and DNA damage repair [22]. Two distinct pathways that synthesize dNTPs are the de novo synthesis in the cytoplasm, and the salvage pathway that takes place both in cytoplasm and mitochondria. The rate-limiting step of de novo dNTP synthesis is catalyzed by ribonucleotide reductase (RNR) that converts ribonucleotide diphosphates to deoxyribonucleotides [3]. Degradation of dNTPs as part of the salvage pathway is accomplished by deaminases and phosphorylases, as well as the mammalian triphosphohydrolase, SAMHD1 [7, 8]. Optimization of dNTP pools is achieved by cell cycle-dependent activity and allosteric regulation of RNR and SAMHD1 [23]. Actively proliferating cells have an approximately 10-fold higher dNTP pool than quiescent cells that are in $G_{0} / G_{1}$ phase [24]. The dNTP pool is greatly expanded during $G_{1}$ to $\mathrm{S}$-phase transition, and remains abundant until DNA synthesis is complete [24]. This biphasic regulation is critical to supply dNTPs for DNA synthesis, and to prevent excess intracellular dNTPs in the absence of DNA replication, which can contribute to innate immune activation [25] and cancer development [22].

\section{Dysregulation of dNTP in cancer development}

The complement of intracellular dNTPs has numerous implications for DNA replication, mutagenesis, DNA repair, and therefore in cancer development. Recent progress in literature suggests that RNR-mediated increase in $\mathrm{dNTP}$ pools is accompanied by higher mutation rates due to reduced fidelity of DNA replication or activation of translesion synthesis [26]. These studies suggest that increased dNTP pools upon altered RNR activity may cause increased mutation rates. However, it is important to note that altered RNR activity can also affect its function in DNA repair (25). Therefore, further studies are required to rule out the possibility of altered DNA repair functions of RNR leading to increased mutation rate. Indeed, dNTP pools are generally greater in transformed cell lines compared to normal cells [27]. Mutator phenotypes are characterized by increased somatic mutation frequency in pre-cancerous cells that accounts for high number of mutations in cancer cells, consistent with what is observed with dNTP pool imbalances [24, 28, 29]. These pre-cancerous cells are characterized by enhanced mutagenesis, stimulation of genetic recombination, increased frequency of chromosomal abnormalities, DNA strand breaks and cell death [26]. Imbalance in cellular dNTP pool causes a hypermutator phenotype, associated with DNA replication stress and altered replication fork velocity $[3,11]$. Mechanisms of mutagenesis conferred by imbalanced dNTP pool are not fully understood, but likely include nucleotide misinsertion during DNA replication, indirect inhibition of proof-reading, or forced frameshift mutations [26].

In response to DNA damage, dNTP levels in S-phase increase approximately by 4-fold [30]. Both RNR and SAMHD1 can be recruited to sites of DNA damage to tightly regulate the dNTPs supplied for DNA repair machinery $[11,30]$. Replication stress from increased mutation rate contributes to genomic instability and activates the DNA damage response, which then may further exacerbate mutagenesis by altering the balance of the dNTP pool $[31,32]$. It is interesting to note that precancerous cells show evidence of increased DNA damage response activation [31,32]. Together, these studies suggest that in cells with elevated activation of DNA damage responses, increased dNTP levels may lead to malignancy.

Nucleotide metabolism plays an important role in senescence and autophagy in cancer cells. Senescence may occur as a tumor suppressive mechanism early on in tumor initiation, while autophagy is a common mechanism that tumor cells use to survive under metabolic stress [3, 33]. Nucleotide metabolism therefore has implications in genomic instability and mutation as part of tumor initiation, and resistance to apoptosis during tumor promotion, two of the important hallmarks of 
cancer development. Thus, regulators of dNTP pool are important targets of cancer therapy.

\section{Current cancer therapy targeting dNTP metabolism}

Given the important role of nucleotide metabolism in cell proliferation, transformation and tumor progression, inhibition of nucleotide synthesis has been commonly used in treatment of cancer, as well as infectious and immune-mediated diseases [3]. Inhibiting DNA precursor synthesis or incorporation of nucleoside analogs into DNA results in DNA damage and stalled replication forks, followed by activation of the S-phase checkpoint, which may lead to cell death by apoptosis [3] (Fig. 1). Methotrexate and similar compounds that inhibit dihydrofolate reductase required for purine biosynthesis [34], are widely used in chemotherapy for solid and lymphocytic tumors. On the other hand, purine and pyrimidine analogs such as forodesine and gemcitabine respectively, can disrupt DNA replication [35]. Moreover, 5-fluorouracil is a pyrimidine adduct that is misincorporated into DNA and inhibits nucleotide synthesis [34]. Most of clinically available nucleoside analogs act by incorporation into DNA, which relies on balanced dNTP pool for high fidelity of incorporation during replication. Excess intracellular dNTPs may out-compete these nucleoside analogs, thus conferring chemotherapeutic resistance [36]. For this reason, these drugs may be used in combination with other therapies, such as inhibitors of RNR that reduce dNTP pool. Efficient RNR inhibition is accomplished by hydroxyurea, a free radical scavenger and iron chelator that inactivates the catalytic capacity of the R2 subunit of RNR [3]. Current clinically used inhibitors of RNR, such as hydroxyurea or gemcitabine, have limitations, including severe cytotoxicity, short half-life, and drug resistance when used as a single therapy. New horizons for nucleotide synthesis-directed therapy include

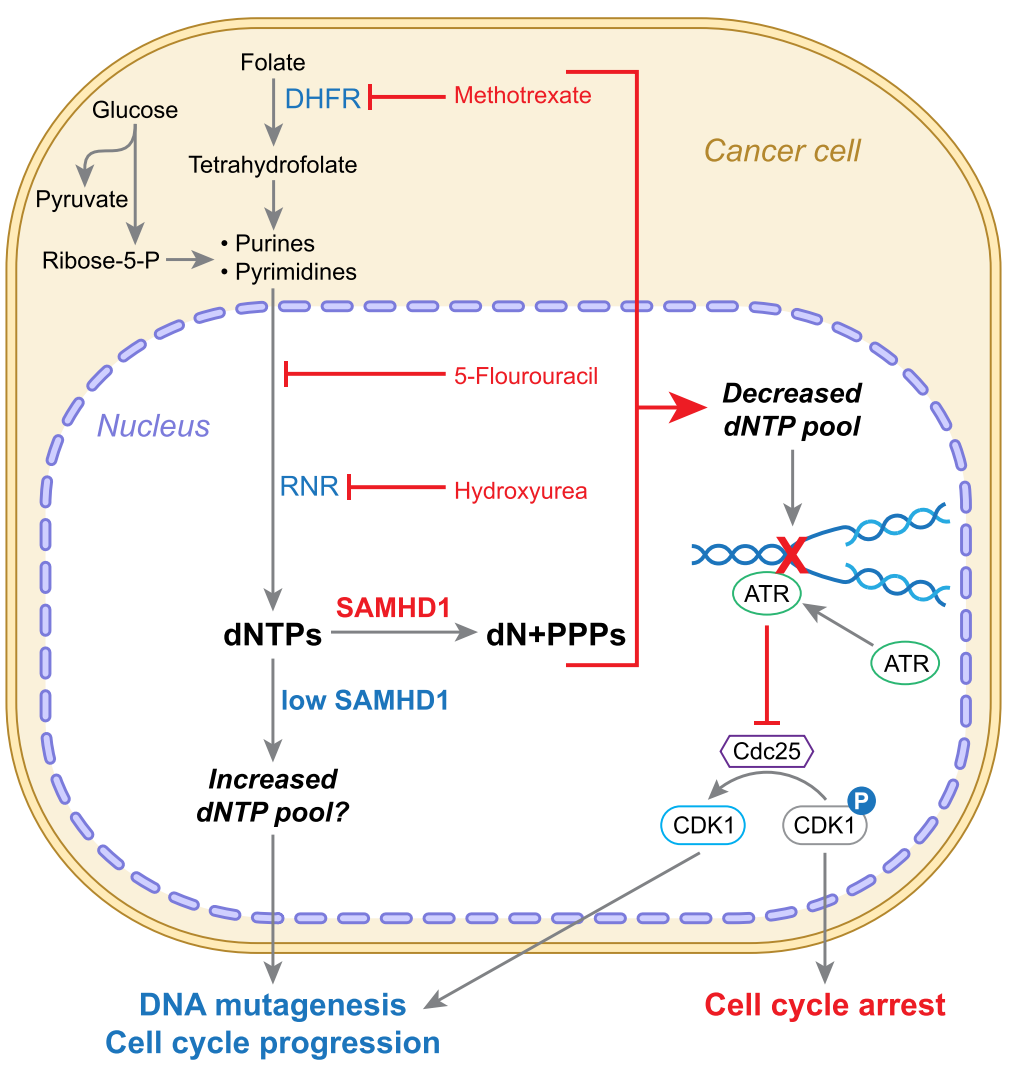

Fig. 1 Dysregulation of dNTPs in cancer pathogenesis and its targeted therapy. Nucleotides are derived from multiple intracellular sources, including products of glycolysis, folate cycle, and scavenging of degraded components. The reduction of dihydrofolate to active tetrahydrofolate is inhibited by the chemotherapeutic methotrexate. Pyrimidine and purine bases are both reduced to deoxynucleosides (dN) by ribonucleotide reductase (RNR). This reaction is inhibited by the chemotherapeutic hydroxyurea. Other steps in this reaction are inhibited by numerous nucleoside analogs ("antimetabolite" compounds) including 5-fluorouracil. These drugs function by limiting the deoxynucleoside triphosphate (dNTP) pool available for DNA synthesis and triggering the S-phase checkpoint via the action of ATR and Chk1, resulting in cell cycle arrest by inhibiting the activation of cyclin dependent kinase 1 (CDK1). A potentially critical regulator of this pathway is SAMHD1, which hydrolyses dNTPs into products that are then recycled or degraded. By this action, SAMHD1 limits dNTP pool in G1 phase and prevents DNA replication. With loss of function or repression of SAMHD1 expression, the dNTP pool is not reduced which can result in DNA damage and inappropriate cell cycle progression. DHFR, dihydrofolate reductase; PPPs, triphosphate; ATR, ataxia-telangiectasia and Rad3-related protein; Cdc25, cell division cycle 25 
small molecule targeting subunit activity modulation, such as anti-sense oligonucleotides and gene therapy targeting RNR $[3,26]$.

It is currently unclear whether SAMHD1 can degrade these nucleoside analogs in cells and reduce their anticancer potency. A study indicates that reduction of SAMHD1 levels in T-cells significantly decreases HIV-1 sensitivity to thymidine analogs (such as zidovudine or stavudine), but not other tested nucleotide analogs of HIV-1 reverse transcriptase inhibitors [37]. These results suggest that SAMHD1 may have a differential effect over the different dNTPs. Owing to its dNTPase activity, SAMHD1 may serve as an intriguing target of cancer therapy as reduction of dNTP pool may prevent tumorigenesis or an increase in efficacy of anti-nucleotide chemotherapeutics (Fig. 1).

\section{Regulation of nucleotide metabolism by dNTP hydrolysis} Regulating dNTP homeostasis

SAMHD1 was identified in human myeloid cells as a novel HIV-1 restriction factor [5, 6]. SAMHD1 is responsible for maintaining low levels of intracellular dNTPs in non-dividing cells $[9,38,39]$. In non-transformed fibroblasts, knockdown of SAMHD1 results in loss of cellcycle regulation, and cells accumulate in $\mathrm{G}_{1}$ with oversized dNTP pool [40]. Therefore, SAMHD1 influences cell cycle progression and DNA replication by degrading dNTPs [40] (Fig. 1). SAMHD1 siRNA-mediated knockdown has differential effects on the dNTP pools of proliferating cells and quiescent cells [40]. In proliferating lung or skin fibroblasts, siRNA-mediated knockdown of SAMHD1 leads to loss of the cell-cycle regulation of dNTP pool sizes and thus resulting in dNTP imbalance [40]. On the other hand, in cells made quiescent via serum starvation, SAMHD1 down-regulation leads to a marked expansion of dNTP pools [40]. The function of SAMHD1 in sensing intracellular dNTPs is critical for maintaining pool balance in cells at the appropriate phase in cell cycle.

The opposite role of increasing the intracellular dNTP pool is achieved by RNR, which catalyzes the rate-limiting step in de novo nucleotide synthesis by converting ribonucleotides to deoxyribonucleotides [3, 24]. RNR heterotetramer is present at consistent levels throughout the cell cycle and functions in de novo nucleotide synthesis even in non-dividing cells [27]. The gene encoding the active subunit, R1, is transcriptionally regulated, responding to DNA replication stress and progression through the cell cycle [31]. RNR transcription increases by 10-20 fold in S phase over the levels in G1 phase [27, 31]. RNR can also be recruited to sites of DNA damage, by the virtue of p53inducible subunit R2, to supply the necessary dNTPs for the damage repair machinery [27]. Cancer cells require RNR for de novo synthesis of dNTPs and elevated RNR expression is a characteristic of many cancers, which contribute to increased dNTP levels and uncontrolled cellular proliferation [27].

Both SAMHD1 and RNR have similar regulation mechanisms of their expression and activity, which are cell-cycle dependent and tightly controlled in cells. Both enzymes are active oligomers with two types of allosteric sites that sense the concentration of nucleotides in the cell and control enzymatic activity [23, 24, 41]. Regulation of SAMHD1 dNTPase activity is achieved by allosteric binding of dGTP or GTP to the first allosteric site, followed by binding of any dNTP to the second allosteric site, causing a conformational change in the substrate-binding pocket $[23,42]$. SAMHD1 has sensory activity that detects the cellular dNTP pool composition, and allows a feedback system to achieve a balance with the opposite catalytic activity of RNR [23, 41]. The most sensitive regulator of dNTP pool is likely dATP, as it has higher affinity for the second allosteric site of SAMHD1, weak affinity for the catalytic site of SAMHD1, and is produced least efficiently by RNR [26, 43]. The dATP:ATP ratio is likewise an important regulator of RNR activity [26]. Elevations in dATP concentrations are inhibitory to the anabolic activity of RNR, whereas increased ATP concentration is stimulatory [26]. Therefore, dATP plays a finetuned regulatory role in providing negative feedback from SAMHD1 to RNR and vice versa [23, 26, 41, 43]. Allosteric binding of dNTPs is responsible for tetramerization of SAMHD1 to its functional catalytic state. Tetramerdisrupting mutations abolish dNTPase activity as well as the ability of SAMHD1 to restrict HIV-1 infection [23, 42].

\section{Restricting viral infection}

SAMHD1 inhibits retroviral replication in non-dividing cells by depleting the intracellular dNTP pool to a level that is limiting for viral reverse transcription $[9,38,39]$. In terminally differentiated macrophages and dendritic cells, or resting $\mathrm{CD}^{+}{ }^{+} \mathrm{T}$-cells with arrested cell cycle $\left(G_{0} / G_{1}\right)$, dNTP levels are significantly decreased by SAMHD1, and therefore HIV-1 infection is restricted $[9,38,39]$. Vpx, a protein encoded by HIV-2 or certain simian immunodeficiency viruses, causes proteasomal degradation of SAMHD1, which is associated with an increase in dNTP pool, accelerated proviral DNA synthesis, and enhanced viral infectivity $[9,38,39]$. Low cellular dNTP serves as a common mechanism of SAMHD1-mediated retroviral restriction. In addition to HIV-1, SAMHD1 also restricts many other retroviruses and DNA viruses (such as herpes simplex virus 1 and hepatitis B virus) in non-dividing cells [44-46]. However, it remains unclear how these viral restriction studies provide any clues that may link with cell cycle dysregulation in cancer cells, particularly in the context of viral infection. 
Interaction with cell-cycle regulatory proteins

Phosphorylation at threonine (T) 592 by cyclin dependent kinases (CDK) regulates dNTPase activity and HIV-1 restriction activity of SAMHD1 [47-49]. CDK1 as well as CDK2 in complex with cyclin A can bind to and phosphorylate this site [25, 47-49]. CDK2 interaction with SAMHD1 is regulated by CDK6 in Tcells and macrophages [50]. Type I IFN reduces phosphorylation of SAMHD1 at the T592 residue [47], likely by inducing $C D K$ inhibitors, such as p21, resulting in HIV-1 restriction and decreased dNTP pool $[50,51]$. For proper maintenance of dNTP levels, SAMHD1 protein must be reduced in the cell during $\mathrm{S}$-phase to facilitate DNA replication [40]. In differentiated human macrophages, SAMHD1 interacts with cyclin L2, forming an ubiquitin ligase complex and resulting in its proteasomal degradation [52]. However, it is unclear whether the degradation process of endogenous SAMHD1 occurs in other cell types or in dividing cells. Phosphorylation of SAMHD1 by CDKs may be the signal for its proteasomal degradation in a cell type- or cell cycle-dependent manner. With an understanding of SAMHD1 dNTPase function and $\mathrm{dNTP}$ regulation in cancer, we discuss the physiological consequences of alterations in SAMHD1 expression.

\section{SAMHD1 alterations in an autoimmune disease and cancers}

\section{Mutations of SAMHD1 in an autoimmune disease}

SAMHD1 is a negative regulator of IFN-induced innate immune responses [53]. Aicardi-Goutieres syndrome (AGS) is an hereditary neurodegenerative autoimmune disorder that is characterized by progressive encephalopathy and is accompanied by increased IFN- $\alpha$ production [54]. AGS is attributed to defective clearance of excessive intracellular (self) nucleic acids, which trigger immune responses resulting in activation of IFN pathway in a manner that mimics viral infection [10, 55]. SAMHD1 homozygous mutations have been identified in $17 \%$ of patients with AGS, suggesting a pivotal role of SAMHD1 in preventing activation of innate immune response to selfnucleic acids [10]. Further, a homozygous deletion ( 9 $\mathrm{Kb})$ in the $S A M H D 1$ gene was identified in patients with atypical AGS, characterized by genomic instability [56]. Mutations in $S A M H D 1$ result in increased dNTP pools in fibroblasts from AGS patients [25], suggesting that the dNTPase function of SAMHD1 prevents autoimmunity by maintaining genome stability. Together, these studies implicate SAMHD1 in regulation of self-nucleic acid stimulated-autoimmune responses.

\section{Mutations of SAMHD1 in cancers}

One of the most critical mechanisms by which cancer cells grow uncontrollably is by inhibiting the function of tumor suppressor genes [57]. This is achieved mainly through mutation (leading to loss of function) and/or downregulation of tumor suppressor genes that normally arrest cell growth [57]. Studies using CLL patient samples have identified $S A M H D 1$ mutations in 4 out of 160 cases (2.5\%) [13]. Acquired SAMHD1 mutations were reported at a frequency of $11 \%$ in relapsed or chemotherapy refractory CLL patients and $3 \%$ in the pretreatment group [11]. These findings suggest that SAMHD1 mutation can be an important driving factor contributing to CLL progression, and can potentially be utilized as a biomarker for CLL prognosis.

SAMHD1 somatic mutations have been identified in several human cancers, including chronic lymphocytic leukemia (CLL) [11, 13, 16], myeloma [18], breast cancer [17], lung carcinoma [14], colon and rectal cancer $[17,19,58]$, pancreatic cancer [12], and glioblastoma [15]. All the identified SAMHD1 alterations in cancer are summarized in Table 1. Of note, several of these mutations are located in the catalytic region of HD domain of SAMHD1 that is responsible for its dNTPase activity (Table 1) [7, 8]. However, it is important to note that it is still unknown whether these specific mutations in the HD domain of SAMHD1 can directly affect its dNTPase function. Generation and characterization of SAMHD1 variants harboring these specific mutations can help define their activity changes. Importantly, physiological significance and functional contributions of these $S A M H D 1$ mutations to progression of cancer also needs to be investigated, especially since the $S A M H D 1$ knockout in mice does not result in spontaneous tumorigenesis [53, 59]. Further functional analyses of SAMHD1 mutations identified in human cancer cells would aid in answering these important questions.

\section{Downregulation of SAMHD1 expression in cancers}

$S A M H D 1$ mRNA levels were significantly lower in CLL patient B-cells with $S A M H D 1$ mutation relative to normal B-cells [11]. Although this study suggests that these mutations result in reduction of SAMHD1 mRNA levels, the exact mechanism involved in this process is unknown. It is important to determine whether these mutations lead to reduced transcription of SAMHD1 or if they cause a post-transcriptional change such as nonsense-mediated decay of SAMHD1 mRNA. SAMHD1 expression is also significantly lower in other cancers, including cutaneous T-cell lymphoma (CTCL) [20], lung carcinoma [21], breast carcinomas and various tumor cell lines [11].

CTCL is a subset of non-Hodgkin's lymphomas that is characterized by infiltration and proliferation of malignant $\mathrm{CD}_{4}{ }^{+} \mathrm{T}$-lymphocytes into the skin [60]. Decreased mRNA and protein levels of SAMHD1 were identified in peripheral blood mononuclear cells (PBMCs) of CTCL 
Table 1 Summary of the identified SAMHD1 alterations in various human cancers

\begin{tabular}{|c|c|c|c|c|c|}
\hline \multirow[t]{3}{*}{ Cancers } & \multicolumn{4}{|c|}{ Identified SAMHD1 alterations } & \multirow[t]{3}{*}{ References } \\
\hline & \multicolumn{2}{|l|}{ Gene mutations } & \multirow[t]{2}{*}{ Epigenetic alterations } & \multirow[t]{2}{*}{ Consequences } & \\
\hline & Frequencies & $\begin{array}{l}\text { Amino acid changes } \\
\text { (domain locations) }{ }^{\mathrm{a}}, \mathrm{b}\end{array}$ & & & \\
\hline Breast cancer & $0.4 \%^{\mathrm{c}}(4 / 981$ cases $)$ & N.A. & N.A. & Reduced SAMHD1 protein & {$[11,17]$} \\
\hline Chronic lymphocytic leukemia & $2.5 \%^{\mathrm{a}}[13]$ (4/160 cases) & N.A. & N.A. & $\begin{array}{l}\text { Reduced SAMHD1 mRNA } \\
\text { and protein }\end{array}$ & {$[11,13,16]$} \\
\hline Colorectal cancer & $2.2 \%^{\mathrm{c}}$ (5/223 cases) & $\begin{array}{l}\text { F59C (SAM domain) } \\
\text { D207Y (HD domain) } \\
\text { R226H (HD domain) } \\
\text { T232M (HD domain) } \\
\text { S247Y (HD domain) } \\
\text { K288T (HD domain) }\end{array}$ & N.A. & N.A. & {$[17,19,58]$} \\
\hline Cutaneous T-cell lymphoma & N.A. & N.A. & Promoter DNA methylation & $\begin{array}{l}\text { Reduced SAMHD1 mRNA } \\
\text { and protein }\end{array}$ & [20] \\
\hline Glioblastoma & $0.3 \%^{c}(1 / 290$ cases $)$ & N.A. & N.A. & N.A. & [15] \\
\hline Lung cancer & $1.7 \%^{\mathrm{c}}$ (4/228 cases) & A441T (HD domain) ${ }^{d}$ & Promoter DNA methylation & $\begin{array}{l}\text { Reduced SAMHD1 mRNA } \\
\text { and protein }\end{array}$ & {$[14,21]$} \\
\hline Myeloma & $1 \%^{\mathrm{c}}$ (2/205 cases) & Y521D (HD domain) ${ }^{d}$ & N.A. & N.A. & [18] \\
\hline Pancreatic cancer & $1.1 \%^{c}(1 / 91$ cases $)$ & N.A. & N.A. & N.A. & [12] \\
\hline
\end{tabular}

N.A. Information not available

${ }^{\mathrm{a}}$ The mutation details are based on cited literature

${ }^{b}$ It is unclear whether all of these mutations in the HD domain of SAMHD1 can directly affect its dNTPase function

'The mutation rates are based on TCGA data analysis via cBioportal (http://www.cbioportal.org/public-portal/) [67]

${ }^{\mathrm{d}}$ The amino acid (aa) changes and their positions; the sterile alpha motif (SAM) domain (aa 45-110); the catalytic region (aa 167-311) of the histidine-aspartate (HD) domain (aa 115-562) [68] 
patients relative to healthy donors [20]. However, it is unknown whether decreased SAMHD1 expression has an effect on CTCL lymphomagenesis, if restored SAMHD1 expression can rescue CTCL phenotype, or whether SAMHD1 mRNA/protein expression correlates with patient prognosis. Lung carcinoma patients have reduced SAMHD1 mRNA and protein [21], but a direct correlation to disease progression or outcome has not been established. Overexpression of SAMHD1 in a lung cancer cell line results in decreased proliferation in vitro, with a concomitant increase in intracellular dNTP levels [21]. Similarly, overexpression of SAMHD1, but not the dNTPase-defective mutant, can significantly reduce HeLa cell proliferation [11].

\section{Epigenetic regulation of SAMHD1 in cancers}

Epigenetic mechanisms play a major role in cancer development and progression by modulating gene expression [61]. DNA methylation and chromatin remodeling via histone acetylation or deacetylation are the most important epigenetic mechanisms through which cancer cells achieve increased expression of oncogenes or decreased expression of tumor suppressor genes [61]. Thus, many therapeutic strategies target epigenetic mechanisms in cancer [62].

Studies in lung cancer and CTCL patients have revealed the role of epigenetic mechanisms in downregulation of SAMHD1 expression. The SAMHD1 promoter in PBMCs of CTCL patients is highly methylated, while it is unmethylated in PBMCs from healthy donors [20]. This was also observed in human lymphoma/leukemia $\mathrm{CD} 4^{+} \mathrm{T}$-cell lines relative to primary $\mathrm{CD} 4^{+} \mathrm{T}$-cells that have high endogenous SAMHD1 expression [63]. Importantly, treatment with DNA methyltransferase inhibitors in $\mathrm{CD}^{+}{ }^{+} \mathrm{T}$-cell lines rescues SAMHD1 mRNA and protein expression, validating that DNA methylation has a direct inhibitory effect on SAMHD1 transcription [63]. Similar effects of SAMHD1 promoter methylation on its expression were identified in lung cancer tissues [21]. Interestingly, inhibition of histone deacetylation leads to induction of SAMHD1 mRNA and protein levels in $\mathrm{CD}_{4}^{+} \mathrm{T}$ cells, suggesting that histone deacetylation also contributes to repression of SAMHD1 expression [63]. Despite implications for epigenetic downregulation of SAMHD1 in cancer, the exact mechanisms are not known. Better understanding of these pathways may aid in development of therapeutic strategies targeting epigenetic modifications.

Gene expression is also regulated by microRNAs and transcription factors. Although, transcription factors that can regulate SAMHD1 expression are yet to be identified, one study has indicated that microRNA-181 binds directly to the 3' untranslated region of SAMHD1 mRNA and downregulates its expression [64]. Overexpression of microRNA-181 in monocytic THP-1 cells reduced endogenous SAMHD1 mRNA and protein levels, while microRNA-181 downregulation in T-cell leukemic-derived Jurkat cells lead to an increase in SAMHD1 mRNA and protein [64]. It is unclear whether microRNA-181 downregulates SAMHD1 expression in cancer patients. Together these findings support the hypothesis that SAMHD1, through its dNTPase function, could potentially act as a tumor suppressor (Fig. 2).

\section{SAMHD1 may act as a tumor suppressor by maintaining genome stability}

Cancer cells frequently exhibit genomic instability and are characterized by increased mutation rate, increased incidence of chromosomal rearrangement, and frequent retroelement insertions. Many studies demonstrate that dNTP homeostasis is necessary for maintenance of accurate DNA replication and efficient DNA damage repair [22]. By regulating dNTP homeostasis, SAMHD1 expression has implications in cancer.

SAMHD1 is expressed ubiquitously in undifferentiated and differentiated cell types to regulate DNA damage signaling and facilitate proper activation of the innate immune response [25]. Constitutive DNA damage signaling, as noted in pre-neoplastic cells with genomic instability, is associated with cell cycle delay, senescence, and upregulation of IFN-stimulated genes. AGS patient cells have increased sensitivity to genotoxic stimuli, and respond to DNA damage with increased rates of mutagenesis [10]. However, it remains unclear whether AGS patients with $S A M H D 1$ mutations have a higher predisposition to cancer development. Notably, an AGS patient harboring a germ line mutation in SAMHD1 developed CLL [11]. Loss of SAMHD1 enhances resistance to chemotherapy-induced DNA damage [11]. Following treatment with DNA-damaging agents, SAMHD1 protein is recruited to sites of double-stranded DNA breaks and results in increased cell death, a protective mechanism in cells [11]. In addition to a causal link between DNA damage signaling and innate immune activation, SAMHD1 also prevents aberrant synthesis of DNA species and accumulation of endogenous retroelements that can activate DNA sensors and trigger type I IFN immune responses [10, 25, 53].

Approximately $17 \%$ of the human genome is composed of retrotransposons that may cause genomic insertions, termed long interspersed elements (LINE) [65]. SAMHD1-mediated LINE1 inhibition is through blocking of the reverse transcriptase activity of the element. Moreover, AGS-associated SAMHD1 mutants are defective in inhibiting LINE1 and silencing SAMHD1 in cells results in an increase in retrotransposition activity [65]. Thus, SAMHD1 may play a key role in reducing random insertions by retroelements that contribute to genomic instability and lead to cancer development. 


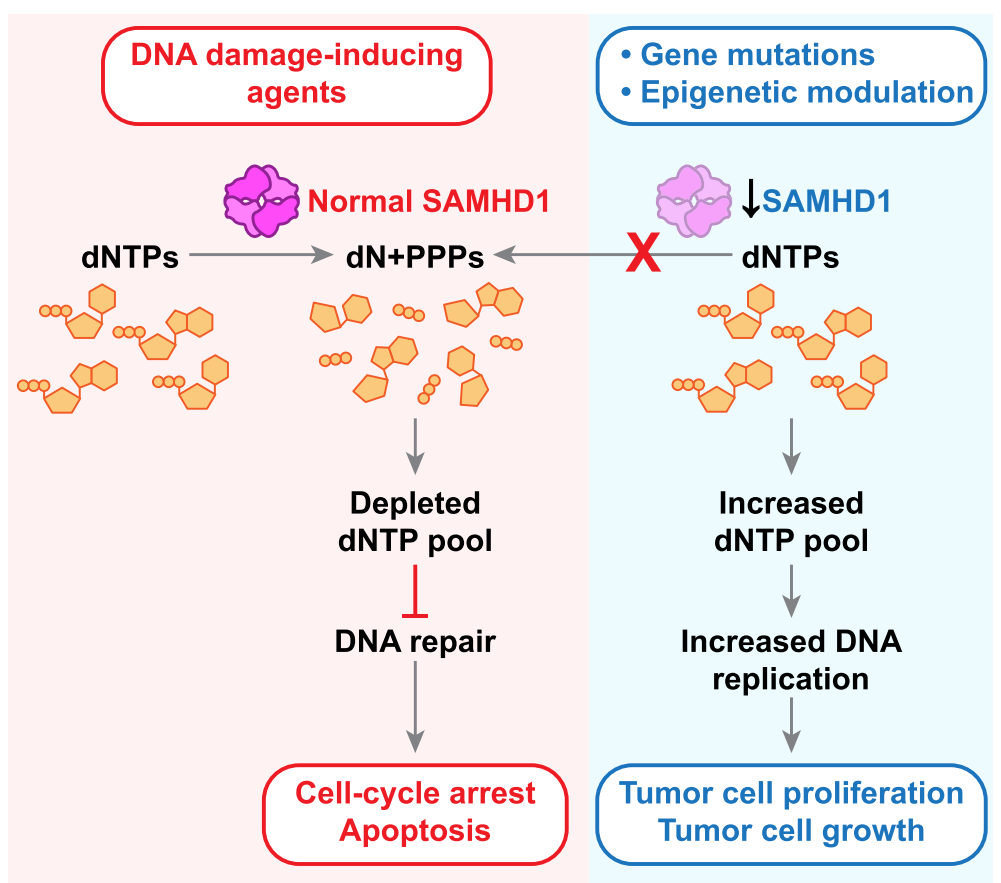

Fig. 2 SAMHD1 may function as a potential tumor suppressor via regulation of dNTPs. SAMHD1 is a novel mammalian dNTP triphosphohydrolase enzyme that helps in maintenance of intracellular dNTP homeostasis. SAMHD1 has been identified to be downregulated in cutaneous T-cell lymphoma and lung cancer patient samples via increased promoter DNA methylation. Downregulated SAMHD1 expression may increase the dNTP pool in these cancers resulting in enhanced DNA replication and thus tumor cell growth and proliferation. On the other hand, overexpression of SAMHD1 protein upon treatment with DNA damage-inducing agents may result in depleted dNTP pools leading to defective DNA damage repair mechanisms and thus cell cycle arrest and apoptosis

A homozygous large deletion in SAMHD1 was detected in patients with atypical AGS featuring multiple mitochondrial DNA deletions [56], highlighting the importance of balanced cytoplasmic dNTP pool affecting mitochondrial pools $[40,56]$. Concentrations of dNTPs are highly correlated between cytoplasm and mitochondria in nontransformed cells [27]. The dNTP levels in both cytoplasm and mitochondria are critical to fidelity of DNA replication, mutagenesis, and genome integrity $[27,66]$. Thus, loss of SAMHD1 activity has functional consequences for dysregulated DNA replication, damage repair, and genomic integrity, thereby contributing to tumorigenesis.

\section{Conclusions and perspectives}

Mutagenesis and disruption of genomic stability are two important factors leading to cancer development, which can be prevented by maintenance of optimal intracellular dNTP pools. Balanced dNTP levels are required for DNA damage repair that is necessary to prevent cancer initiation [22]. Excess intracellular dNTP pool is common in cancer cells, not only conferring proliferative ability to the cell, but also posing a challenge for successful therapy targeting dNTP metabolism. Nucleoside analogs have been a mainstay of cancer treatment for decades [4], however persistently high levels of dNTP in the cell can out-compete the drug and result in cancer resistance. Mutations in SAMHD1 have been suggested to contribute to therapeutic resistance due to a loss of dNTP hydrolysis [11]. Combination therapies that target both the synthetic and hydrolytic pathways of nucleotides would likely result in better outcomes. Additionally, restoration of SAMHD1 by targeting the epigenetic mechanisms using histone deacetylase inhibitors may sensitize cancer cells to DNA damage-inducing therapies, such as treatment with poly (ADP ribose)-polymerase inhibitors. Therefore, it will be important to invest further efforts in understanding the complexities of nucleotide metabolism as it relates to regulation of cell cycle, mutagenesis, and cancer development.

SAMHD1 is a key regulator of dNTP homeostasis via its dNTPase function $[7,8]$ and this activity is important to prevent innate and autoimmune responses [7]. Similarly, dNTPase activity of SAMHD1 could play an antiproliferative role in cancer pathophysiology (Fig. 2). Several studies in different cancers demonstrated SAMHD1 mutation or downregulation, implicating it as a potential tumor suppressor. Restoring SAMHD1 expression in cancer cell lines leads to decreased cell proliferation supporting the hypothesis that SAMHD1 has anti-proliferative effects in transformed cells [11, 21]. Additionally, SAMHD1 
may assist in DNA damage response and contribute to cell cycle regulation (Fig. 2).

Despite rapid progress of structural and functional studies of SAMHD1, its exact role and mechanisms by which it regulates cancer cell growth and proliferation remain to be understood further. In vivo studies of loss of function and expression of SAMHD1 in cancer are lacking. Although mouse models with $S A M H D 1$ knockout have been established [53, 59], these mice do not develop spontaneous cancers at the age of 70-96 weeks [53, 59], indicating that SAMHD1 reduction alone is not sufficient to develop cancer in mice. As with most tumor suppressor genes, it is likely that additional hits are required. Loss or impaired SAMHD1 function could confer partial but significant selective advantage to proliferating cancer cells due to an increase or imbalance in intracellular dNTPs (Fig. 2).

Understanding the mechanisms that downregulate SAMHD1 in cancers is of utmost importance as it could support developing novel strategies to restore SAMHD1 expression in cancer cells and thus reduce tumorigenicity. Epigenetic mechanisms regulate SAMHD1 expression in CTCL and lung cancers [20, 21]. Identifying the detailed mechanisms and molecules that are involved would lead to approaches that restore SAMHD1 to physiologically normal levels. This may enhance the efficacy of the current DNA damage-inducing strategies in cancer treatment that target $\mathrm{dNTP}$ regulation. In conclusion, illumination of the significance of SAMHD1 in cancer development would open up new avenues of discovery in studying dNTP regulation and cancer biology.

\begin{abstract}
Abbreviations
AGS: Aicardi-Goutieres syndrome; CDK: Cyclin-dependent kinase; CLL: Chronic lymphocytic leukemia; CTCL: Cutaneous T cell lymphoma; dNTP: Deoxynucleotide-triphosphate; GTP: Guanosine triphosphate; HIV: Human immunodeficiency virus; IFN: Interferon; LINE: Long interspersed elements; RNR: Ribonucleotide reductase; SAMHD1: Sterile alpha motif and HD-domain containing protein 1; Vpx: Viral protein X.
\end{abstract}

\section{Competing interests}

The authors declare that they have no competing interests.

\section{Authors' contributions}

All the authors conceived the structure and content of the review. RK and KMK have contributed equally to writing the manuscript. All the authors read and approved the manuscript.

\section{Acknowledgments \\ We thank the members of the Wu lab for stimulating discussions. We also thank Mr. Tim Vojt for illustrations presented in this manuscript (reproduced by permission of The Ohio State University). The research in the Wu laboratory was supported by grants (CA181997 and Al104483) to L.W. from the National Institutes of Health and by the program of Public Health Preparedness for Infectious Diseases of The Ohio State University.}

\section{Author details}

${ }^{1}$ Center for Retrovirus Research, Department of Veterinary Biosciences, The Ohio State University, 1900 Coffey Road, Columbus, OH 43210, USA. 2Department of Microbial Infection and Immunity, The Ohio State University, Columbus, $\mathrm{OH} 43210$, USA. ${ }^{3}$ Comprehensive Cancer Center, The Ohio State University, Columbus, $\mathrm{OH}$ 43210, USA.
Received: 19 May 2015 Accepted: 18 September 2015

Published online: 29 September 2015

\section{References}

1. Kunz BA, Kohalmi SE, Kunkel TA, Mathews CK, Mclntosh EM, Reidy JA. International Commission for Protection Against Environmental Mutagens and Carcinogens. Deoxyribonucleoside triphosphate levels: a critical factor in the maintenance of genetic stability. Mutat Res. 1994;318(1):1-64.

2. Reichard P. Interactions between deoxyribonucleotide and DNA synthesis. Annu Rev Biochem. 1988;57:349-74. doi:10.1146/annurev.bi.57.070188.002025.

3. Aird KM, Zhang R. Nucleotide metabolism, oncogene-induced senescence and cancer. Cancer Lett. 2015;356(2 Pt A):204-10. doi:10.1016/j.canlet.2014.01.017.

4. Galmarini CM, Mackey JR, Dumontet C. Nucleoside analogues and nucleobases in cancer treatment. Lancet Oncol. 2002;3(7):415-24.

5. Hrecka K, Hao C, Gierszewska M, Swanson SK, Kesik-Brodacka M, Srivastava S, et al. Vpx relieves inhibition of HIV-1 infection of macrophages mediated by the SAMHD1 protein. Nature. 2011;474(7353):658-61. doi:10.1038/nature10195.

6. Laguette N, Sobhian B, Casartelli N, Ringeard M, Chable-Bessia C, Segeral E, et al. SAMHD1 is the dendritic- and myeloid-cell-specific HIV-1 restriction factor counteracted by Vpx. Nature. 2011;474(7353):654-7. doi:10.1038/nature10117.

7. Powell RD, Holland PJ, Hollis T, Perrino FW. Aicardi-Goutieres syndrome gene and HIV-1 restriction factor SAMHD1 is a dGTP-regulated deoxynucleotide triphosphohydrolase. J Biol Chem. 2011;286(51):43596-600. doi:10.1074/jbc.C111.317628.

8. Goldstone DC, Ennis-Adeniran V, Hedden JJ, Groom HC, Rice Gl, Christodoulou E, et al. HIV-1 restriction factor SAMHD1 is a deoxynucleoside triphosphate triphosphohydrolase. Nature. 2011;480(7377):379-82. doi:10.1038/nature10623.

9. Lahouassa H, Daddacha W, Hofmann H, Ayinde D, Logue EC, Dragin L, et al. SAMHD1 restricts the replication of human immunodeficiency virus type 1 by depleting the intracellular pool of deoxynucleoside triphosphates. Nat Immunol. 2012;13(3):223-8. doi:10.1038/ni.2236.

10. Rice Gl, Bond J, Asipu A, Brunette RL, Manfield IW, Carr IM, et al. Mutations involved in Aicardi-Goutieres syndrome implicate SAMHD1 as regulator of the innate immune response. Nat Genet. 2009;41(7):829-32. doi:10.1038/ng.373.

11. Clifford R, Louis T, Robbe P, Ackroyd S, Burns A, Timbs AT, et al. SAMHD1 is mutated recurrently in chronic lymphocytic leukemia and is involved in response to DNA damage. Blood. 2014;123(7):1021-31. doi:10.1182/blood-2013-04-490847.

12. Jones S, Zhang X, Parsons DW, Lin JC, Leary RJ, Angenendt P, et al. Core signaling pathways in human pancreatic cancers revealed by global genomic analyses. Science. 2008;321(5897):1801-6. doi:10.1126/science.1164368.

13. Landau DA, Carter SL, Stojanov P, McKenna A, Stevenson K, Lawrence MS, et al. Evolution and impact of subclonal mutations in chronic lymphocytic leukemia. Cell. 2013;152(4):714-26. doi:10.1016/j.cell.2013.01.019.

14. Liu J, Lee W, Jiang Z, Chen Z, Jhunjhunwala S, Haverty PM, et al. Genome and transcriptome sequencing of lung cancers reveal diverse mutational and splicing events. Genome Res. 2012;22(12):2315-27. doi:10.1101/gr.140988.112.

15. Parsons DW, Jones S, Zhang X, Lin JC, Leary RJ, Angenendt P, et al. An integrated genomic analysis of human glioblastoma multiforme. Science. 2008;321(5897):1807-12. doi:10.1126/science.1164382.

16. Schuh A, Becq J, Humphray S, Alexa A, Burns A, Clifford R, et al. Monitoring chronic lymphocytic leukemia progression by whole genome sequencing reveals heterogeneous clonal evolution patterns. Blood. 2012;120(20):4191-6. doi:10.1182/blood-2012-05-433540

17. Sjoblom T, Jones S, Wood LD, Parsons DW, Lin J, Barber TD, et al. The consensus coding sequences of human breast and colorectal cancers. Science. 2006;314(5797):268-74. doi:10.1126/science.1133427.

18. Walker BA, Wardell CP, Melchor L, Hulkki S, Potter NE, Johnson DC, et al. Intraclonal heterogeneity and distinct molecular mechanisms characterize the development of $\mathrm{t}(4 ; 14)$ and $\mathrm{t}(11 ; 14)$ myeloma. Blood. 2012;120(5):1077-86. doi:10.1182/blood-2012-03-412981.

19. Seshagiri S, Stawiski EW, Durinck S, Modrusan Z, Storm EE, Conboy CB, et al. Recurrent R-spondin fusions in colon cancer. Nature. 2012;488(7413):660-4. doi:10.1038/nature11282.

20. de Silva S, Wang F, Hake TS, Porcu P, Wong HK, Wu L. Downregulation of SAMHD1 expression correlates with promoter DNA methylation in Sezary syndrome patients. J Invest Dermatol. 2014;134(2):562-5. doi:10.1038/jid.2013.311.

21. Wang JL, Lu FZ, Shen XY, Wu Y, Zhao LT. SAMHD1 is down regulated in lung cancer by methylation and inhibits tumor cell proliferation. Biochem Biophys Res Commun. 2014;455(3-4):229-33. doi:10.1016/j.bbrc.2014.10.153. 
22. Bester AC, Roniger M, Oren YS, Im MM, Sarni D, Chaoat M, et al. Nucleotide deficiency promotes genomic instability in early stages of cancer development. Cell. 2011;145(3):435-46. doi:10.1016/j.cell.2011.03.044.

23. Ji X, Wu Y, Yan J, Mehrens J, Yang H, DeLucia M, et al. Mechanism of allosteric activation of SAMHD1 by dGTP. Nat Struct Mol Biol. 2013;20(11):1304-9. doi:10.1038/nsmb.2692.

24. Rampazzo C, Miazzi C, Franzolin E, Pontarin G, Ferraro P, Frangini M, et al. Regulation by degradation, a cellular defense against deoxyribonucleotide pool imbalances. Mutat Res. 2010;703(1):2-10. doi:10.1016/j.mrgentox.2010.06.002.

25. Kretschmer S, Wolf C, Konig N, Staroske W, Guck J, Hausler M, et al. SAMHD1 prevents autoimmunity by maintaining genome stability. Ann Rheum Dis. 2015;74(3):e17. doi:10.1136/annrheumdis-2013-204845.

26. Aye $Y$, Li M, Long MJ, Weiss RS. Ribonucleotide reductase and cancer: biological mechanisms and targeted therapies. Oncogene. 2015;34(16):2011-21. doi:10.1038/onc.2014.155.

27. Gandhi W, Samuels DC. A review comparing deoxyribonucleoside triphosphate (dNTP) concentrations in the mitochondrial and cytoplasmic compartments of normal and transformed cells. Nucleosides Nucleotides Nucleic Acids. 2011;30(5):317-39. doi:10.1080/15257770.2011.586955.

28. Loeb LA. A mutator phenotype in cancer. Cancer Res. 2001;61(8):3230-9.

29. Venkatesan RN, Bielas JH, Loeb LA. Generation of mutator mutants during carcinogenesis. DNA Repair (Amst). 2006;5(3):294-302. doi:10.1016/ j.dnarep.2005.10.012.

30. Niida H, Shimada M, Murakami H, Nakanishi M. Mechanisms of dNTP supply that play an essential role in maintaining genome integrity in eukaryotic cells. Cancer Sci. 2010;101(12):2505-9. doi:10.1111/j.1349-7006.2010.01719.x.

31. Bartkova J, Horejsi Z, Koed K, Kramer A, Tort F, Zieger K, et al. DNA damage response as a candidate anti-cancer barrier in early human tumorigenesis. Nature. 2005:434(7035):864-70. doi:10.1038/nature03482.

32. Gorgoulis VG, Vassiliou LV, Karakaidos P, Zacharatos P, Kotsinas A, Liloglou T, et al. Activation of the DNA damage checkpoint and genomic instability in human precancerous lesions. Nature. 2005;434(7035):907-13. doi:10.1038/nature03485.

33. Chen W, Zhang L, Zhang K, Zhou B, Kuo ML, Hu S, et al. Reciprocal regulation of autophagy and dNTP pools in human cancer cells. Autophagy. 2014;10(7):1272-84. doi:10.4161/auto.28954.

34. Longley DB, Harkin DP, Johnston PG. 5-fluorouracil: mechanisms of action and clinical strategies. Nat Rev Cancer. 2003;3(5):330-8. doi:10.1038/nrc1074.

35. Ewald B, Sampath D, Plunkett W. Nucleoside analogs: molecular mechanisms signaling cell death. Oncogene. 2008;27(50):6522-37. doi:10.1038/onc.2008.316

36. Yoshio N, Kawai Y, Hori H, Ueda T. Resistance to 9-beta-D-arabinofuranosyl2-fluoroadenine due to reduced incorporation into DNA from competition by excess deoxyadenosine triphosphate: implications for different sensitivities to nucleoside analogues. Int J Hematol. 2005;81(5):405-12.

37. Ballana E, Badia R, Terradas G, Torres-Torronteras J, Ruiz A, Pauls E, et al. SAMHD1 specifically affects the antiviral potency of thymidine analog HIV reverse transcriptase inhibitors. Antimicrob Agents Chemother. 2014:58(8):4804-13. doi:10.1128/AAC.03145-14

38. Baldauf HM, Pan X, Erikson E, Schmidt S, Daddacha W, Burggraf M, et al. SAMHD1 restricts HIV-1 infection in resting CD4(+) T cells. Nat Med. 2012;18(11):1682-7. doi:10.1038/nm.2964.

39. St Gelais C, de Silva S, Amie SM, Coleman CM, Hoy H, Hollenbaugh JA, et al. SAMHD1 restricts HIV-1 infection in dendritic cells (DCs) by dNTP depletion, but its expression in DCs and primary CD4+ T-lymphocytes cannot be upregulated by interferons. Retrovirology. 2012;9:105. doi:10.1186/1742-4690-9-105.

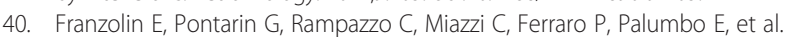
The deoxynucleotide triphosphohydrolase SAMHD1 is a major regulator of DNA precursor pools in mammalian cells. Proc Natl Acad Sci U S A. 2013;110(35):14272-7. doi:10.1073/pnas.1312033110.

41. Nordlund P, Reichard P. Ribonucleotide reductases. Annu Rev Biochem. 2006;75:681-706. doi:10.1146/annurev.biochem.75.103004.142443.

42. Zhu C, Gao W, Zhao K, Qin X, Zhang Y, Peng X, et al. Structural insight into dGTPdependent activation of tetrameric SAMHD1 deoxynucleoside triphosphate triphosphohydrolase. Nat Commun. 2013;4:2722. doi:10.1038/ncomms3722.

43. Ji X, Tang C, Zhao Q, Wang W, Xiong Y. Structural basis of cellular dNTP regulation by SAMHD1. Proc Natl Acad Sci U S A. 2014;111(41):E4305-14. doi:10.1073/pnas.1412289111.

44. Gramberg T, Kahle T, Bloch N, Wittmann S, Mullers E, Daddacha W, et al. Restriction of diverse retroviruses by SAMHD1. Retrovirology. 2013;10:26. doi:10.1186/1742-4690-10-26.
45. Kim ET, White TE, Brandariz-Nunez A, Diaz-Griffero F, Weitzman MD. SAMHD1 restricts herpes simplex virus 1 in macrophages by limiting DNA replication. J Virol. 2013;87(23):12949-56. doi:10.1128/JVI.02291-13.

46. Chen Z, Zhu M, Pan X, Zhu Y, Yan H, Jiang T, et al. Inhibition of Hepatitis B virus replication by SAMHD1. Biochem Biophys Res Commun. 2014:450(4):1462-8. doi:10.1016/j.bbrc.2014.07.023.

47. Cribier A, Descours B, Valadao AL, Laguette N, Benkirane M. Phosphorylation of SAMHD1 by cyclin A2/CDK1 regulates its restriction activity toward HIV-1. Cell Rep. 2013;3(4):1036-43. doi:10.1016/j.celrep.2013.03.017.

48. White TE, Brandariz-Nunez A, Valle-Casuso JC, Amie S, Nguyen LA, Kim B, et al. The retroviral restriction ability of SAMHD1, but not its deoxynucleotide triphosphohydrolase activity, is regulated by phosphorylation. Cell Host Microbe. 2013;13(4):441-51. doi:10.1016/j.chom.2013.03.005.

49. St Gelais C, de Silva S, Hach JC, White TE, Diaz-Griffero F, Yount JS, et al. Identification of cellular proteins interacting with the retroviral restriction factor SAMHD1. J Virol. 2014;88(10):5834-44. doi:10.1128/ JVI.00155-14.

50. Pauls E, Ruiz A, Badia R, Permanyer M, Gubern A, Riveira-Munoz E, et al. Cel cycle control and HIV-1 susceptibility are linked by CDK6-dependent CDK2 phosphorylation of SAMHD1 in myeloid and lymphoid cells. J Immunol. 2014;193(4):1988-97. doi:10.4049/jimmunol.1400873.

51. Pauls E, Ruiz A, Riveira-Munoz E, Permanyer M, Badia R, Clotet B, et al. p21 regulates the HIV-1 restriction factor SAMHD1. Proc Natl Acad Sci U S A. 2014;111(14):E1322-4. doi:10.1073/pnas.1322059111.

52. Kyei GB, Cheng X, Ramani R, Ratner L. Cyclin L2 is a critical HIV dependency factor in macrophages that controls SAMHD1 abundance. Cell Host Microbe. 2015;17(1):98-106. doi:10.1016/j.chom.2014.11.009.

53. Behrendt R, Schumann T, Gerbaulet A, Nguyen LA, Schubert N, Alexopoulou D, et al. Mouse SAMHD1 has antiretroviral activity and suppresses a spontaneous cell-intrinsic antiviral response. Cell Rep. 2013;4(4):689-96. doi:10.1016/j.celrep.2013.07.037

54. Goutieres F. Aicardi-Goutieres syndrome. Brain Dev. 2005;27(3):201-6. doi:10.1016/j.braindev.2003.12.011

55. Lee-Kirsch MA. Nucleic acid metabolism and systemic autoimmunity revisited. Arthritis Rheum. 2010;62(5):1208-12. doi:10.1002/art.27372.

56. Leshinsky-Silver E, Malinger G, Ben-Sira L, Kidron D, Cohen S, Inbar S, et al. A large homozygous deletion in the SAMHD1 gene causes atypical AicardiGoutieres syndrome associated with mtDNA deletions. Eur J Hum Genet. 2011;19(3):287-92. doi:10.1038/ejhg.2010.213.

57. Hanahan D, Weinberg RA. Hallmarks of cancer: the next generation. Cell. 2011;144(5):646-74. doi:10.1016/j.cell.2011.02.013.

58. Network CGA. Comprehensive molecular characterization of human colon and rectal cancer. Nature. 2012;487(7407):330-7. doi:10.1038/ nature 11252.

59. Rehwinkel J, Maelfait J, Bridgeman A, Rigby R, Hayward B, Liberatore RA, et al. SAMHD1-dependent retroviral control and escape in mice. EMBO J. 2013;32(18):2454-62. doi:10.1038/emboj.2013.163.

60. Wong HK, Mishra A, Hake T, Porcu P. Evolving insights in the pathogenesis and therapy of cutaneous T-cell lymphoma (mycosis fungoides and Sezary syndrome). Br J Haematol. 2011;155(2):150-66. doi:10.1111/j.13652141.2011.08852.x.

61. Baylin SB, Jones PA. A decade of exploring the cancer epigenome biological and translational implications. Nat Rev Cancer. 2011;11(10):726-34. doi:10.1038/nrc3130.

62. Hagelkruys A, Sawicka A, Rennmayr M, Seiser C. The biology of HDAC in cancer: the nuclear and epigenetic components. Handb Exp Pharmacol. 2011:206:13-37. doi:10.1007/978-3-642-21631-2_2.

63. de Silva S, Hoy H, Hake TS, Wong HK, Porcu P, Wu L. Promoter methylation regulates SAMHD1 gene expression in human CD4+ T cells. J Biol Chem. 2013;288(13):9284-92. doi:10.1074/jbc.M112.447201.

64. Jin C, Peng X, Liu F, Cheng L, Lu X, Yao H, et al. MicroRNA-181 expression regulates specific post-transcriptional level of SAMHD1 expression in vitro. Biochem Biophys Res Commun. 2014;452(3):760-7. doi:10.1016/j.bbrc.2014.08.151

65. Zhao K, Du J, Han X, Goodier JL, Li P, Zhou X, et al. Modulation of LINE-1 and Alu/SVA retrotransposition by Aicardi-Goutieres syndromerelated SAMHD1. Cell Rep. 2013;4(6):1108-15. doi:10.1016/ j.celrep.2013.08.019.

66. Kumar D, Viberg J, Nilsson AK, Chabes A. Highly mutagenic and severely imbalanced dNTP pools can escape detection by the S-phase checkpoint. Nucleic Acids Res. 2010;38(12):3975-83. doi:10.1093/nar/gkq128. 
67. Cerami E, Gao J, Dogrusoz U, Gross BE, Sumer SO, Aksoy BA, et al. The CBio cancer genomics portal: an open platform for exploring

multidimensional cancer genomics data. Cancer Discov. 2012;2(5):401-4. doi:10.1158/2159-8290.CD-12-0095.

68. Wu L. Cellular and Biochemical Mechanisms of the Retroviral Restriction Factor SAMHD1. ISRN Biochem. 2013;2013. doi:10.1155/2013/728392.

Submit your next manuscript to BioMed Central and take full advantage of:

- Convenient online submission

- Thorough peer review

- No space constraints or color figure charges

- Immediate publication on acceptance

- Inclusion in PubMed, CAS, Scopus and Google Scholar

- Research which is freely available for redistribution 